\title{
Asthma und Rhinitis - eine Erkrankung, eine Therapie
}

D ie allergische Rhinitis ist eine der häufigsten immunologischen Störungen, die enge Verwandschaft mit Asthma bronchiale und Sinusitis rührt von gemeinsamen pathophysiologischen Mechanismen der oberen und unteren Atemwege her. „Die nasale Mukosa und die Bronchialschleimhaut sind sehr ähnlich", sagte Jean Bousquet (Montpellier) auf dem 20. EAACIKongress in Berlin. „Die einzigen Unterschiede sind, dass die Nase stärker arteriell durchblutet wird und im Gegensatz zur Lunge keine Muskelzellen besitzt". Eine allergische Entzündung verläuft in beiden Organen gleich: Entzündungszellen wie Makrophagen, Mastzellen und Eosinophile setzen Arachidonsäure frei. Die daraus entstehenden Leukotriene (LT) erhöhen die Gefäßpermeabilität und die Schleimsekretion, verursachen nasale Obstruktion und Bronchokonstriktion; sie stören die Funktion des Flimmerepithels und locken weitere Eosinophile an. „Eine kurze Allergen-Exposition kann zu einer 24 Stunden lang nachweisbaren Entzündung führen“, erklärte G. Walter Canonica (Genua). Denn ein einziges Molekül LTD4 wirkt $1000 \mathrm{mal}$ stärker als Histamin. Die Dosis-abhängige Bronchokonstriktion beginnt später, hält aber länger an als nach Histamin-Provokation.

\section{Das Picknick im Park ist kein Tabu mehr}

„Es besteht kein Zweifel mehr über die Wirksamkeit der Leukotrien-Antagonisten beim allergischen Asthma bronchiale“, sagte Sergio Bonini (Rom). Doch Leukotriene spielen auch bei anderen atopischen Erkrankungen wie Rhinitis, Konjunktivitis und allergischen Hautkrankheiten eine bedeutende Rolle. Eli Meltzer (San Diego) berichtete über die „Day-in-the-Park“-Studie [Donnelly AL et al. Am J Resp Crit Care Med 1995; 151: 1734-9], bei der Patienten mit allergischer Rhinitis zwei Frühlingstage im Park verbrachten. Dabei induzierten bereits $20 \mathrm{mg}$ des Leukotrien-Antagoni- sten Zafirlukast eine signifikante Verbesserung der Symptome Niesen, Rhinorrhö und nasale Obstruktion im Gegensatz zu Plazebo. Neuere Studien zeigten im Vergleich zu Plazebo eine signifikante Besserung von Tages- und Nachtbeschwerden sowie Lebensqualität durch Montelukast, wobei $10 \mathrm{mg}$ Montelukast in Kombination mit $10 \mathrm{mg}$ Loratadin den Einzelsubstanzen überlegen war.

Bei den verschiedenen allergischen Manifestationen eines Patienten stellen Leukotriene den „gemeinsamen Nenner" dar. Die oralen, systemisch wirkenden Leukotrien-Antagonisten ermöglichen eine einheitliche Therapie, die an verschiedenen Zielorten greift. In Kombination mit Antihistaminika wirken die Substanzen additiv. Steroide hingegen nehmen keinen Einfluss auf den Leukotrien-Spiegel. Zur Behandlung der allergischen Rhinitis empfiehlt Eli Meltzer Leukotrien-Antagonisten als Monotherapie oder in Kombination mit Antihistaminika bzw. intranasale Steroide.

Symposium „Asthma and other allergic disorders: overlapping mediators with a common treatment?", EAACI-Kongress, Berlin 2001. Veranstalter: MSD

\section{Rekombinante Primärprävention}

D e subkutane spezifische Immuntherapie (SIT) gilt bis heute neben der Allergenkarenz als die einzige kausale Therapie bei IgE-vermittelten allergischen Erkrankungen. Risikopatienten zu erkennen und mit hochspezifischen Allergenextrakten präventiv zu behandeln, ist daher nach den Worten von Prof. Harald Renz, Marburg, die große Herausforderung für die allergologische Forschung in den kommenden Jahren.

Zur Behandlung empfahl Prof. Renz ein synergistisches Therapiekonzept, das auf die individuelle Situation des Patienten abgestimmt ist und eine Balance zwischen antientzündlicher Pharmakotherapie und Hyposensibilisierung darstellt. Wie effektiv letztere aber ist, hängt von der Qualität der ein- gesetzten Allergene ab. Heute sind eine Reihe gut charakterisierter und standardisierter Allergenextrakte auf dem Markt, die über eine hohe Sicherheit verfügen.

In der näheren Zukunft könnten aber Allergenextrakte zur Verfügung stehen, die ebenso sicher sind wie die heute gebräuchlichen, jedoch ohne die bekannten Nebenwirkungen wie Sofortreaktionen an den Mastzellen. Die ALKSCHERAX Arzneimittel GmbH arbeitet gegenwärtig mit Hochdruck an der Entwicklung gentechnisch modifizierter Allergene, die diesem Anspruch entsprechen sollen.

Das Konzept besteht in einer kontrollierten Reduktion der IgE-Bindung an das Allergen durch gentechnische Verän- derungen in den dominierenden IgE-Epitopen, ohne dass die dreidimensionale Struktur des Allergens verändert wird. Damit bleibt die IgG-Antwort als Reaktion auf das natürliche Allergen erhalten mit dem Vorteil des Ausbleibens von $\mathrm{Ne}$ benwirkungen. Als Beispiel präsentierte Dr. Larsen das am besten erforschte und charakterisierte Birkenpollen-Allergen BET v 1. Induzierte Punktmutationen in Bereichen, die für die Th2-Zell-vermittelte überschießende Immunreaktion verantwortlich sind, führten bereits in vitro $\mathrm{zu}$ ersten Erfolgen. Aufgrund der Sequenz-Homologie von BET v 1 zu anderen Allergenen soll der künftige Impfstoff auch bei Patienten wirksam sein, die z. B. allergisch auf Äpfel, Haselnuss, Sellerie oder Karotten reagieren.

„German Allergy Symposium“, Hørsholm 2001. Veranstalter: ALK SCHERAX 